\title{
Coal petrographic genetic units in deltaic-plain deposits of the Campanian Mesa Verde Group (New Mexico, USA)
}

\author{
N. Buillit ${ }^{a}$ E. Lallier-Vergès ${ }^{a}$, B. Pradier ${ }^{b}$ and G. Nicolas ${ }^{b}$ \\ a Institut des Sciences de la Terre d'Orléans, Université d'Orléans, 45067 Orléans Cedex 2, \\ France \\ ${ }^{\mathrm{b}}$ TotalFinaElf-CSTJF/Pau, Avenue Larribau, 64018 Pau Cedex, France
}

Author Keywords: Mesa Verde group; Menefee formation; Genetic stratigraphy; Coal; Maceral analysis; Microlithotype

\begin{abstract}
The Campanian rocks of the Mesa Verde Group in the San Juan Basin, New Mexico, USA, form a littoral wedge composed of offshore, shoreface and onshore facies. This siliciclastic wedge was interpreted as a landward-to-seaward stepping of grouped genetic units, i.e. intermediate term cycles. The continental facies consist of coastal-plain deposits (coals, shales, and sandstones) represented by three main domains, progressively distant from the palaeoshoreline: the deltaic-plain, the intermediate-plain, and the alluvial-plain domains. In the deltaic-plain, grouped aggradational-retrogradational genetic units consist of preserved coal-shale doublets alternating with thick shales. Although previous works have identified genetic units in the shoreface facies, the existence of the latter has not been proven in the coastal-plain deposits. As coal-shale doublets may represent such genetic units, an organic petrography study was performed at high resolution on an $80-\mathrm{cm}$ thick, $1.5-\mathrm{km}$ long coal seam to demonstrate this hypothesis. In this work, the depositional environments of the initial peatlands were rebuilt utilizing two complementary methods: maceral and microlithotype analyses. The results reveal the existence of internal sequences in the coal seam, corresponding to recurrent patterns of a palaeogeographical model proposed for a littoral domain. The authors interpret this coal sequence as the record of successive progradational and aggradational events. Furthermore, a previous study of the organic content of the shales located above the coal demonstrated that the preservation of these facies is related to higher accommodation/peat production and accommodation/sedimentary supply ratios. Coal-shale doublets are then interpreted as elemental genetic units of the deltaic-plain formation. This study demonstrates that, contrary to original concepts, littoral coal seams are not just markers of stable shoreline conditions at a high time scale, but also record landscape migration.
\end{abstract}




\section{Introduction, global setting and objectives}

Coal seams preserved in littoral settings are often used as lithostratigraphic markers of shoreline stability. Undeniably, precursor peats require stable environmental conditions to develop, accumulate, and be preserved as coal beds. More precisely, the occurrence and the geometry of these littoral coals are related to the Base Level fluctuations Powell, 1897), i.e. to the balance between accommodation (space created or suppressed in the area considered), sedimentary supply, and peat production rate (e.g., Cross; Diessel and Bohacs). The Campanian wedge of the Mesa Verde Group is made up of fluvio-deltaic sediments that outcrop within extensive canyons in the San Juan Basin (Colorado/New Mexico, USA). The series consists of sandstones, shales, and relatively thin coal beds $(<1 \mathrm{~m})$. These outcrops provide a great opportunity to study in detail the location and the features of littoral coal seams in their stratigraphic context.

The Mesa Verde sediments were deposited along the littoral environments of the Western Interior Cretaceous Seaway, the epicontinental sea that crossed the American continent from Alaska to the Gulf of Mexico during the Cretaceous (Kauffman, 1977; Fig. 1a). They were subsequently preserved in the Western Interior Cretaceous Basin (Fig. 1b) that formed during the subduction of the Pacific plate beneath the North American plate. The subduction created compressive forces on the Colorado Plateau and led to the Sevier and Laramide orogenies (Woodward and Cross). Owing to the Sevier orogeny, eastward thrusting and uplift of the Rocky Mountains occurred, and thrust-sheet loading of the continental crust caused greater subsidence along the western basin margin (Cross, 1986). As a result of low subsidence to the east, the Western Interior Basin is asymmetric in cross-section ( Kauffman, 1977; Fig. 1b). The uplift of the Laramide orogeny then segmented the seaway into smaller basins, in which the Cretaceous through Tertiary strata are exposed today (Weimer, 1960). In the San Juan Basin, the Cretaceous series are made up of regressive Dakota Sandstone followed by three progradational-retrogradational events (Fig. 1c): the Gallup Sandstone, the Mesa Verde Group and the Pictured Cliff Sandstone. The Mesa Verde Group consists of five lithostratigraphic units (Fig. 1c): the Mancos Shale (marine formation), the Point Lookout Sandstone (shoreface formation), the Menefee Formation (coastal-plain formation), the Cliff House Sandstone (shoreface formation), and the Lewis Shale (marine formation). A major transgressive event called Turnaround separates the Lower Menefee Formation and the Upper Menefee Formation (Eschard and Navarre). This sedimentary series corresponds to a 3-4 Ma regressive-trangressive cycle (Cobban et al., 1994). In the lower progradational section, the Mancos Shale, the Point Lookout Sandstone, and the Lower Menefee Formations are timeequivalent units, whereas in the retrogradational section the time-equivalent units are the Upper Menefee Formation, the Cliff House Sandstone, and the Lewis Shale.

In the previous work based on the Genetic Stratigraphy theory (Cross and Homewood), Navarre (1995) interpreted the Mesa Verde wedge in terms of differential preservation of landscapes due to variations of accommodation/sedimentary supply ratio $(A / S)$. Based on the Walther's (1893-1894) law, Genetic Stratigraphy postulates that an elemental variation in the Base level generates an elemental regressive-transgressive cycle called a genetic unit. 


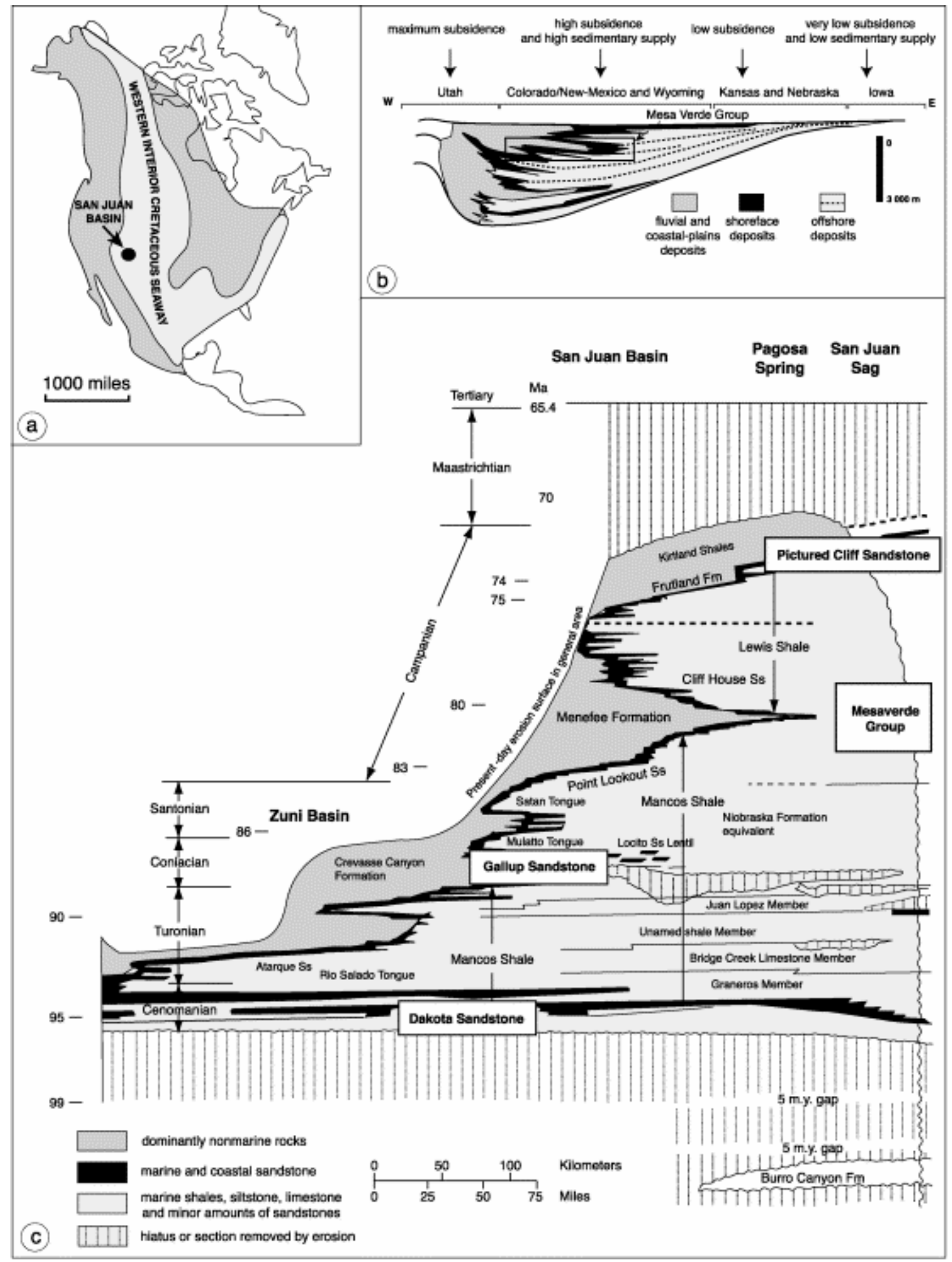

Fig. 1. General geological setting. (a) Map of the Western Interior Cretaceous Seaway (adopted from Kauffman, 1977). (b) Cross-section of the Western Interior Cretaceous Basin (adopted from Kauffman, 1977). (c) General stratigraphic architecture of the Mesa Verde Group (adopted from Navarre and Molenaar).

The regressive, i.e. progradational, part of the genetic unit corresponds to a Base Level Fall episode $(A / S<1)$ and is represented by a downward pointing triangle (see legend of Fig. 2) whereas its transgressive, i.e., retrogradational part, corresponds to the Base Level Rise episode $(A / S>1)$ and is represented by an upward pointing triangle. If an aggradational episode is recorded $(A / S=1)$, it is represented by a rectangle. Cycles can be defined at different time scales and subsequently be fitted together (genetic units are summed into grouped 
genetic units, which in turn are summed into a long-term cycle). Focusing on the sandstone facies of the Mesa Verde, Navarre (1995) defined progradational-retrogradational grouped genetic units in shoreface and coastal-plain formations. But the author failed to precisely relate cycles from both domains. More recently, a new sedimentological model based on the distribution of the organic facies in the Menefee Formation has been proposed (Buillit et al., submitted; Fig. 2). The model suggests the existence of three coastal-plain domains, progressively distant from the palaeoshorelines in the Menefee Formation: the deltaic-plain, the intermediate-plain, and the alluvial-plain. This led to a new definition of the grouped genetic units in the three coastal-plain domains, in terms of aggradational-retrogradational cycles. In the deltaic-plain deposits, the aggradational part of these grouped genetic units consists of sequences of three to five coal seams, continuous over about $10 \mathrm{~km}$, whereas their retrogradational part consists of thick shale facies with isolated coal beds. At a higher scale, coal layers of the aggradational part of the cycle are separated by shales (Fig. 2). In the Lower Menefee Formation, these coal-shale doublets increase in thickness, whereas their thickness decreases in the Upper Menefee Formation. The recurrence of the doublets within the whole deltaic-plain facies suggests the existence of high-scale cycles, i.e. genetic units. But the sedimentological study failed to reveal evidence of their existence.

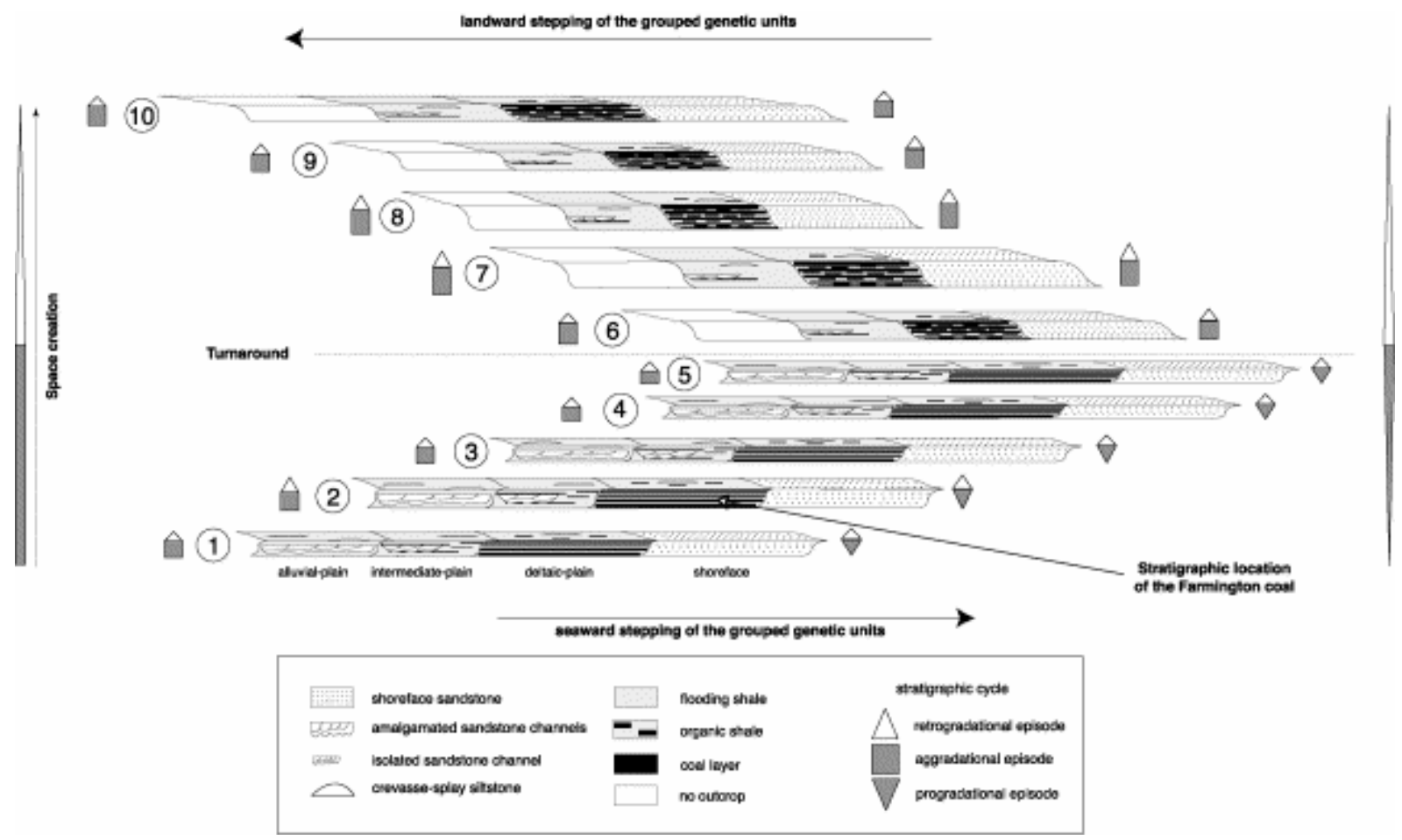

Fig. 2. Sedimentological model illustrating the seaward to landward stepping of shoreface and coastal-plain units, as well as the sediment volumetric partitioning (Buillit et al., submitted).

The aim of the present work is to search for such evidence in a coal-shale doublet, and then to accurately characterise the supposed cycles in terms of balance between accommodation, sedimentary supply, and peat production rate. The authors have attempted to achieve this objective by means of a high-resolution petrographic study performed on an 80-cm thick, 1.5$\mathrm{km}$ long continuous coal bed which is one of the layers of a grouped genetic unit. 


\section{Outcrop, samples and methods}

The study area is located in northern New Mexico, near the town of Farmington (Fig. 3a). The outcrop is made up of successive monocline hills that dip $45^{\circ}$ eastward (Fig. 3b). The contact between the Point Lookout Sandstone and the Menefee Formation can be observed along the base of the first monocline hill, close to the main road. At this point, the lower part of the Menefee Formation is laterally visible over nearly $2 \mathrm{~km}$; it comprises a series of coal beds separated by shale facies, with interbedded siltstones and sandstones. From a stratigraphic viewpoint, this succession represents the aggradational part of the first grouped genetic unit defined in the Farmington area (Fig. 3c). The present work focused on the second coal bed of the coal series, an 80 -cm thick layer informally named Farmington coal by the authors.

Thirty-three samples were gathered from the Farmington coal (Fig. 3d) at locations chosen so as to allow analysis of both vertical and lateral trends. Samples were systematically taken from the base, middle, and top of the seam. Three petrographic analyses were performed on each sample: maceral analyses, microlithotype, and vitrinite reflectance measurement ( $\mathrm{Rr} \%)$.

(1) Maceral analysis (International and Taylor) consists of identifying and quantifying the components in the coal (i.e., macerals; Stopes, 1935). On the basis of this analysis, Diessel (1986) proposes the use of two parameters to characterise the depositional environments of Permian coals from the Sidney Basin: the Tissue Preservation Index (TPI) and the Gelification Index (GI). A TPI-versus-GI diagram makes it possible to rebuild the following environments: terrestrial, dry forest, reed moor, limnic, wet forest, and swamp forest.

(2) The microlithotype analysis (International and Taylor) consists of determining and quantifying the maceral associations (Seyler, 1931). Hacquebard and Donaldson (1969) defined four microlithotype groups, each corresponding to one environment. They used these groups to represent a double triangle diagram of coal environment settings, slightly modified by Marchioni (1980). More recently, Nicolas et al. (1998) adapted both environmental diagrams (Diessel and Hacquebard-Donaldson/Marchioni) to a study on North Sea Brent coals (Middle Jurassic). In addition, the authors proposed to associate a single depositional environment for each sample, taking into account both analyses (Fig. 4), making these diagrams compatible. The lower triangle of the Hacquebard-Donaldson/Marchioni diagram, which takes into account the mineral fraction of the coals, leads to the definition of two additional environments: swamp and limnic-lagoonar. 


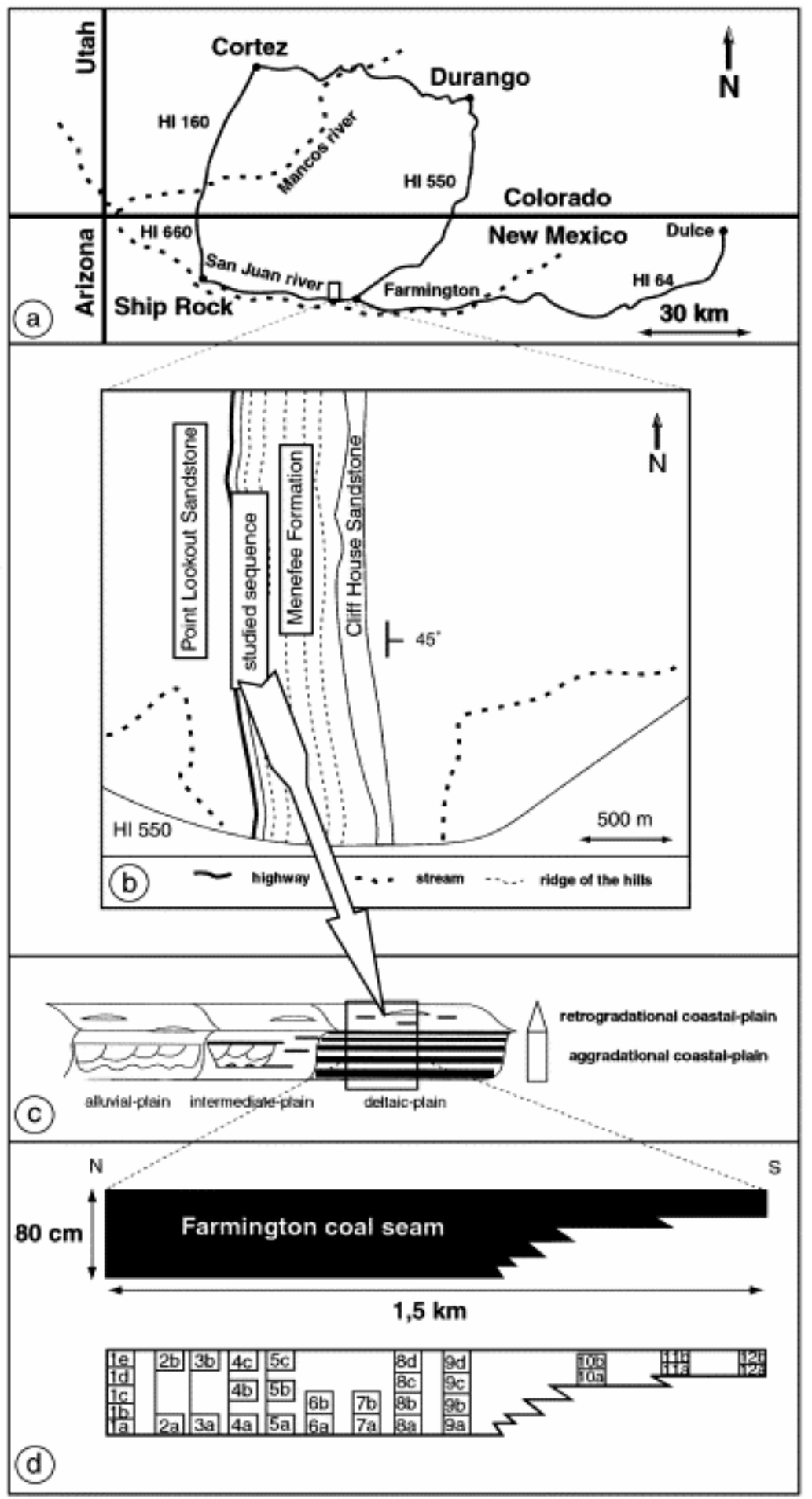

Fig. 3. Diagrams showing: (a) location of the outcrop, New Mexico State; (b) map of the Farmington outcrop; (c) stratigraphic context of the Farmington coal; (d) simplified geometry of the Farmington coal and sample location 


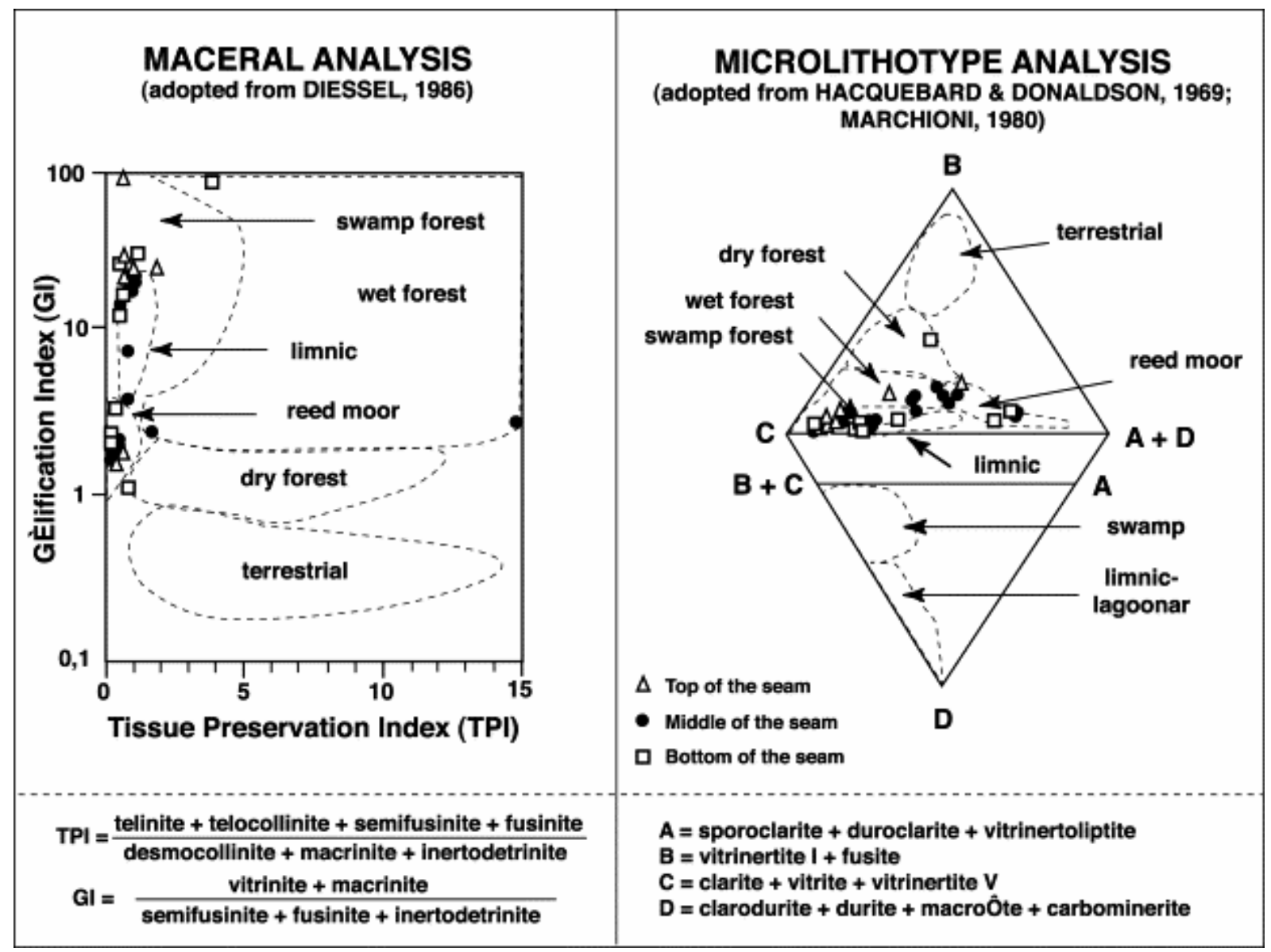

Fig. 4. Location of the depositional environments in the Diessel (left) and the Hacquebard-Donaldson/Marchioni (right) diagrams, modified by Nicolas et al. (1998).

(3) The vitrinite reflectance measurements (Rr\%) are usually performed to evaluate the rank of the organic matter, i.e. its thermal maturity. Depending on the values obtained, one can estimate the position of the strata analysed relative to the oil window in the basin. However, some authors have shown that fluctuations in $\mathrm{Rr} \%$ can also be attributed to coal composition and/or redox conditions during depositional and diagenetic processes (e.g., Gentzis and Ruau).

\section{Petrographic results}

The average maceral composition of the coal bed (Fig. 5, Table 1) consists of an abundant vitrinite group (76\%), mainly made up of desmocollinite (43\%, Fig. 5a), telocollinite (18\%, Fig. 5a), and telinite (15\%). Minor amounts of gelinite, corpocollinite, and vitrodetrinite have been observed. The inertinite group (14\%) is essentially composed of inertodetrinite (7\%, Fig. 5a), semifusinite (4\%, Fig. 5a), and fusinite (3\%, Fig. 5c). Sclerotinite, macrinite, and micrinite are rare. The liptinite group (9.5\%) includes liptodetrinite (4\%), sporinite (3\%, Fig. 5b), resinite (1.5\%, Fig. 5b), and cutinite 1\%, Fig. 5d). 

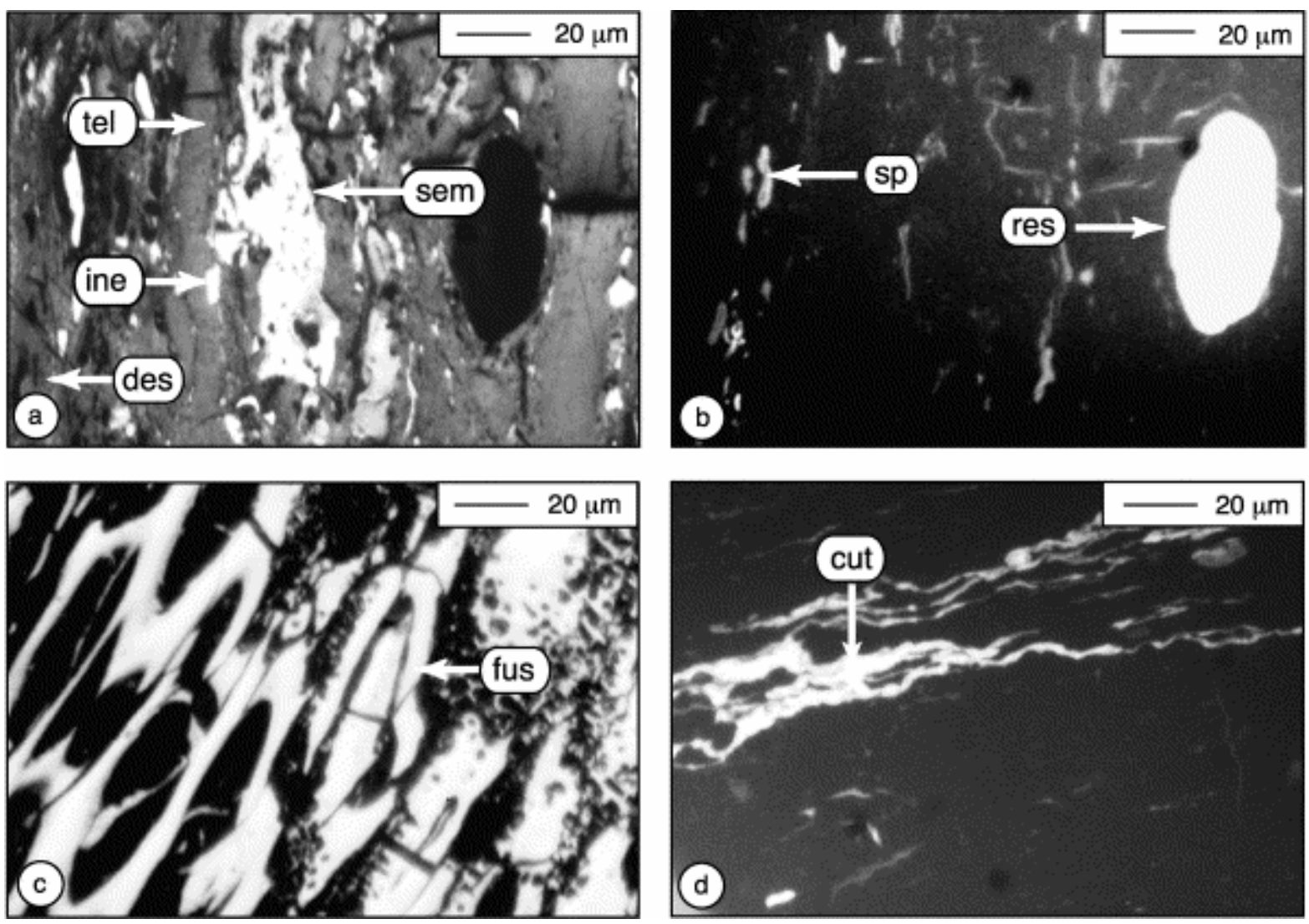

Fig. 5. Photographs of macerals observed with incident light (natural light for the left photographs and UV excitation for the right ones); TEL=telinite, DES=desmocolinite, SEM=semifusinite, FUS=fusinite, INE=inertodetrinite, $\mathrm{SP}=$ spore-pollen, $\mathrm{RES}=$ resinite, $\mathrm{CUT}=$ cutinite .

Table 1. Mean, minimum and maximum values (\%) of maceral groups and macerals (the mineral fraction is not taken into account)

\begin{tabular}{llll}
\hline Maceral & Mean & Minimum & Maximum \\
\hline Vitrinite (\%) & 76.5 & 49.5 & 96.5 \\
Telinite & 15 & 0.5 & 46 \\
Telocollinite & 18 & 2.5 & 68 \\
Desmocollinite & 43.5 & 17 & 60 \\
Corpocollinite & 0.1 & 0 & 2 \\
Vitrodetrinite & 0.03 & 0 & 0.6 \\
Gelinite & 0.15 & 0 & 2.5 \\
& & & \\
Liptinite (\%) & 9.5 & 1.5 & 22 \\
Sporinite & 3 & 0 & 7 \\
Cutinite & 1 & 0 & 4 \\
Resinite & 1.5 & 0 & 5.5 \\
Liptodetrinite & 4 & 1 & 11.25 \\
& & & \\
Inertinite (\%) & 14 & 0.5 & 49 \\
Fusinite & 3 & 0 & 17.5 \\
Semifusinite & 3.75 & 0 & 14.5 \\
Macrinite & 0.01 & 0 & 0.5 \\
Micrinite & 0.01 & 0 & 0.2 \\
Sclerotinite & 0.5 & 0 & 9 \\
Inertodetrinite & 6.75 & 0 & 20 \\
\hline
\end{tabular}


The microlithotypes (Table 2) are mainly represented by monomacerites: vitrite (30\%) and inertite (5\%) bimacerites: clarite (23\%) and vitrinertite V (11\%), and trimacerites: duroclarite (23\%). Mineral associations (carbominerite) are generally poorly represented (2\%).

Table 2. Mean, minimum and maximum values (\%) of microlithotypes

\begin{tabular}{lclc}
\hline Microlithotypes & Mean & Minimum & Maximum \\
\hline Monomacerites & & & \\
Vitrite & 30.25 & 3.5 & 60 \\
Inertite & 5 & 0 & 26.5 \\
Liptite & 0.03 & 0 & 0.5 \\
Bimacerites & & & \\
Vitrinertite V & 11 & 0.5 & 29.5 \\
Vitrinertite I & 2.5 & 0 & 9 \\
Durite & 0.25 & 0 & 3.75 \\
Clarite & 23 & 0 & 50.75 \\
Sporoclarite & 1.5 & 0 & 8.5 \\
& & & \\
Trimacerites & 23 & 0 & 61.5 \\
Duroclarite & 1.25 & 0 & 7.5 \\
Clarodurite & 0.5 & 0 & 3.25 \\
Vitrinertoliptite & & & 12.5 \\
\hline Carbominerites & 2 & 0 & \\
\hline
\end{tabular}

The results of the maceral analysis (Table 3) and the microlithotype study (Table 4) were plotted in the Diessel and Hacquebard-Donaldson/Marchioni diagrams (Fig. 4), taking into account the sample location in the coal seam, i.e., at the bottom, in the middle, or at the top. Although the depositional environments were defined for a given formation (North Sea, Middle Jurassic, Nicolas et al., 1998), 88\% of the samples show good comparison between the Diessel and Hacquebard-Donaldson/Marchioni diagrams (Table 5). The remaining 12\% of samples were determined allowing for the more distinct environment. The results reveal the existence of five depositional environments (reed moor, limnic, swamp forest, wet forest, and dry forest) predominated by the first three (90\%). Considering the Diessel diagram, the mean TPI value for the samples is about 0.84 (excluding samples 1a and 1b) with low standard deviations. The TPI expresses the ratio between mechanically and texturally preserved organic compounds, and those which are not (Diessel, 1992). Judging from the TPI values, depositional conditions indicate poor preservation in the peat. The relatively high amount of desmocollinite and inertodetrinite may indicate extensive transportation of particles within the environments. Contrary to the TPI values, the mean GI value and its standard deviations vary considerably within the Farmington coal. Even though only very minor differences are observed on the log-scale plot of the samples, the calculation of G1 value variations from the base through the middle and to the top of the coal give the following results: $G I=36, G I=31$, GI $=44$ and $\sigma=63, \sigma=15, \sigma=59$. The GI expresses the ratio between gelified and oxidised compounds, indicating substantial variations in humidity conditions (climatic or eustatic) within the peat. The standard deviation values reflect maximum heterogeneity for the 
environments preserved at base of the seam, and maximum homogeneity for the environments preserved in the middle of the seam.

Table 3. Detailed numerical results of the maceral analysis (add "inite" to obtain the complete maceral's names)

\begin{tabular}{|c|c|c|c|c|c|c|c|c|c|c|c|c|c|c|c|c|c|c|c|}
\hline $\mathrm{Nb}$ & Tel. & Telocoll. & Desmocoll. & Corpocoll. & Vitrodet. & Gel. & V (\%) & Spor. & Cut. & Res. & Liptodetr. & L (\%) & Fus. & Semifus. & Macr. & Micr. & Sclerot. & Incrtodet. & I $(\%)$ \\
\hline la & 11.71 & 67.80 & 17.07 & 0.00 & 0.00 & 0.00 & 96.58 & 0.00 & 0.98 & 0.98 & 0.98 & 2.94 & 0.00 & 0.00 & 0.00 & 0.00 & 0.00 & 0.48 & 0.48 \\
\hline lb & 0.48 & 14.01 & 57.97 & 0.00 & 0.00 & 0.00 & 72.46 & 0.48 & 0.00 & 5.31 & 3.86 & 9.65 & 2.89 & 5.79 & 0.48 & 0.00 & 0.00 & 8.69 & 17.85 \\
\hline lc & 2.36 & 15.57 & 48.58 & 0.00 & 0.00 & 0.00 & 66.51 & 2.36 & 0.00 & 2.83 & 4.25 & 9.44 & 1.89 & 5.19 & 0.00 & 0.00 & 1.89 & 15.09 & 24.06 \\
\hline $1 d$ & 0.93 & 9.35 & 56.07 & 0.00 & 0.00 & 0.00 & 66.35 & 1.87 & 0.00 & 2.80 & 4.21 & 8.88 & 6.07 & 4.21 & 0.00 & 0.00 & 0.00 & 14.19 & 24.47 \\
\hline le & 1.39 & 17.21 & 48.37 & 0.00 & 0.00 & 0.00 & 66.97 & 0.00 & 0.47 & 4.65 & 3.26 & 8.38 & 7.44 & 3.26 & 0.00 & 0.00 & 1.39 & 12.56 & 24.65 \\
\hline $2 a$ & 12.87 & 11.88 & 50.00 & 0.00 & 0.00 & 0.00 & 74.75 & 4.46 & 1.49 & 1.98 & 4.46 & 12.39 & 3.47 & 4.46 & 0.00 & 0.00 & 0.00 & 4.95 & 12.88 \\
\hline $2 b$ & 25.12 & 12.81 & 47.29 & 0.00 & 0.00 & 0.00 & 85.22 & 6.89 & 1.48 & 1.48 & 4.43 & 14.28 & 0.00 & 0.00 & 0.00 & 0.00 & 0.49 & 0.00 & 0.49 \\
\hline $3 a$ & 1.47 & 6.37 & 53.43 & 0.00 & 0.00 & 0.00 & 61.27 & 4.41 & 1.47 & 4.90 & 11.27 & 22.05 & 1.96 & 1.96 & 0.00 & 0.00 & 0.98 & 12.25 & 17.15 \\
\hline $3 \mathrm{~b}$ & 33.76 & 8.97 & 39.32 & 0.00 & 0.00 & 0.00 & 82.05 & 5.98 & 3.42 & 1.71 & 4.27 & 15.38 & 0.00 & 0.43 & 0.00 & 0.00 & 0.00 & 2.14 & 2.57 \\
\hline $4 a$ & 42.79 & 12.02 & 34.13 & 0.00 & 0.00 & 0.00 & 88.94 & 3.37 & 0.96 & 0.00 & 4.33 & 8.66 & 0.00 & 0.96 & 0.00 & 0.00 & 0.48 & 0.96 & 2.40 \\
\hline $4 b$ & 11.69 & 4.33 & 40.26 & 0.00 & 0.00 & 0.00 & 56.28 & 3.89 & 0.43 & 4.76 & 8.66 & 17.74 & 7.79 & 11.69 & 0.00 & 0.00 & 0.87 & 5.63 & 25.98 \\
\hline $4 \mathrm{c}$ & 26.05 & 10.23 & 42.79 & 0.00 & 0.00 & 0.00 & 79.07 & 5.58 & 1.86 & 1.86 & 9.77 & 19.07 & 0.93 & 0.00 & 0.00 & 0.00 & 0.00 & 0.93 & 1.86 \\
\hline $5 \mathrm{a}$ & 29.17 & 9.72 & 42.59 & 0.00 & 0.00 & 0.46 & 81.94 & 2.31 & 0.46 & 0.93 & 6.94 & 10.64 & 4.17 & 0.46 & 0.00 & 0.00 & 0.00 & 2.78 & 7.41 \\
\hline $5 b$ & 7.14 & 2.38 & 45.71 & 0.00 & 0.00 & 0.00 & 55.23 & 6.67 & 1.43 & 1.90 & 8.57 & 18.57 & 2.86 & 3.33 & 0.00 & 0.00 & 0.00 & 20.00 & 26.19 \\
\hline $5 \mathrm{c}$ & 27.31 & 15.74 & 38.43 & 0.00 & 0.00 & 0.00 & 81.48 & 3.70 & 4.17 & 0.93 & 6.94 & 15.74 & 0.46 & 0.93 & 0.00 & 0.00 & 0.00 & 1.39 & 2.78 \\
\hline $6 a$ & 15.87 & 15.38 & 50.96 & 0.00 & 0.00 & 0.00 & 82.21 & 5.77 & 0.48 & 0.48 & 5.77 & 12.50 & 0.96 & 0.96 & 0.00 & 0.00 & 0.96 & 2.40 & 5.28 \\
\hline $6 b$ & 27.65 & 15.67 & 42.86 & 0.00 & 0.00 & 0.92 & 87.10 & 3.69 & 1.38 & 0.46 & 4.15 & 9.68 & 0.00 & 1.38 & 0.00 & 0.00 & 0.00 & 1.84 & 3.22 \\
\hline $7 \mathrm{a}$ & 0.00 & 6.37 & 59.80 & 0.00 & 0.00 & 0.00 & 66.17 & 2.94 & 0.49 & 1.96 & 4.90 & 10.29 & 0.49 & 4.90 & 0.00 & 0.00 & 0.00 & 18.14 & 23.53 \\
\hline $7 \mathrm{~b}$ & 6.54 & 41.12 & 41.59 & 0.00 & 0.00 & 0.00 & 89.25 & 2.80 & 0.00 & 1.40 & 2.34 & 6.54 & 0.47 & 3.27 & 0.00 & 0.00 & 0.00 & 0.47 & 4.21 \\
\hline $8 a$ & 21.39 & 22.39 & 47.76 & 0.00 & 0.00 & 0.00 & 91.54 & 1.49 & 0.49 & 0.99 & 3.48 & 6.45 & 0.49 & 0.00 & 0.00 & 0.00 & 0.00 & 1.49 & 1.98 \\
\hline $8 b$ & 4.23 & 7.04 & 52.58 & 0.00 & 0.00 & 0.00 & 63.85 & 3.29 & 0.94 & 0.47 & 2.82 & 7.52 & 4.23 & 7.98 & 0.00 & 0.00 & 0.47 & 15.96 & 28.64 \\
\hline $8 c$ & 5.24 & 15.24 & 45.71 & 0.00 & 0.00 & 0.00 & 66.19 & 3.81 & 0.48 & 0.00 & 5.24 & 9.53 & 12.86 & 4.76 & 0.00 & 0.00 & 0.48 & 5.24 & 23.34 \\
\hline $8 d$ & 13.30 & 7.79 & 36.69 & 0.00 & 0.00 & 0.00 & 57.78 & 3.21 & 0.00 & 1.83 & 1.83 & 6.87 & 10.09 & 5.96 & 0.00 & 0.00 & 0.00 & 17.89 & 33.94 \\
\hline $9 \mathrm{a}$ & 3.19 & 5.48 & 41.09 & 0.00 & 0.00 & 0.00 & 49.76 & 0.00 & 0.00 & 0.00 & 1.37 & 1.37 & 17.35 & 14.61 & 0.00 & 0.00 & 0.00 & 16.89 & 48.85 \\
\hline $9 b$ & 8.52 & 15.69 & 43.49 & 0.00 & 0.00 & 0.00 & 67.70 & 0.45 & 0.00 & 0.45 & 1.35 & 2.25 & 7.17 & 8.52 & 0.00 & 0.00 & 8.97 & 13.45 & 38.11 \\
\hline 9 & 31.90 & 10.48 & 30.48 & 0.00 & 0.00 & 0.00 & 72.86 & 2.86 & 0.48 & 1.43 & 1.90 & 6.67 & 5.24 & 6.19 & 0.00 & 0.00 & 0.00 & 9.05 & 20.48 \\
\hline $9 \mathrm{~d}$ & 46.00 & 19.00 & 31.50 & 0.00 & 0.00 & 0.00 & 96.50 & 1.00 & 0.00 & 1.00 & 1.50 & 3.50 & 0.00 & 1.00 & 0.00 & 0.00 & 0.00 & 2.50 & 3.50 \\
\hline $10 \mathrm{a}$ & 12.38 & 37.14 & 43.33 & 0.00 & 0.00 & 0.48 & 93.33 & 0.48 & 0.48 & 0.00 & 2.38 & 2.34 & 0.00 & 0.00 & 0.00 & 0.00 & 0.00 & 3.33 & 3.33 \\
\hline $10 \mathrm{~b}$ & 17.54 & 33.65 & 40.28 & 0.00 & 0.00 & 0.00 & 91.47 & 0.95 & 0.95 & 0.95 & 2.37 & 5.22 & 0.00 & 1.42 & 0.00 & 0.00 & 0.00 & 1.89 & 3.31 \\
\hline 11a & 23.56 & 18.75 & 44.71 & 0.00 & 0.00 & 2.40 & 89.42 & 2.40 & 0.00 & 0.00 & 0.96 & 3.36 & 1.44 & 1.92 & 0.00 & 0.00 & 0.00 & 3.85 & 7.21 \\
\hline $11 \mathrm{~b}$ & 13.88 & 40.19 & 37.79 & 0.00 & 0.00 & 0.00 & 91.86 & 0.48 & 1.91 & 0.00 & 2.39 & 4.78 & 0.48 & 0.48 & 0.00 & 0.00 & 0.00 & 2.39 & 3.35 \\
\hline $12 \mathrm{a}$ & 3.30 & 26.50 & 48.80 & 0.80 & 0.60 & 0.00 & 80.00 & 3.50 & 1.00 & 1.40 & 1.60 & 7.50 & 0.00 & 7.70 & 0.00 & 0.20 & 0.20 & 1.20 & 9.30 \\
\hline $12 \mathrm{~b}$ & 1.00 & 31.80 & 38.80 & 2.20 & 0.50 & 0.00 & 74.30 & 3.20 & 0.70 & 1.20 & 1.20 & 6.30 & 0.00 & 11.40 & 0.00 & 0.00 & 1.00 & 2.70 & 15.10 \\
\hline
\end{tabular}


Table 4. Detailed numerical results of the microlithotype study

\begin{tabular}{|c|c|c|c|c|c|c|c|c|c|c|c|c|}
\hline Sample & Vitrite & Liptite & Fusite & Clarite & Sporoclarite & Durite & Vitrinertite V & Vitrinertite I & I Duroclarite & Clarodurite & Vitrinertoliptite & Carbominerite \\
\hline la & 60.38 & 0.00 & 0.00 & 11.79 & 8.49 & 0.00 & 6.60 & 0.00 & 0.00 & 0.00 & 0.00 & 12.74 \\
\hline $1 \mathrm{~b}$ & 18.06 & 0.00 & 6.48 & 9.26 & 0.00 & 0.46 & 28.24 & 3.24 & 29.63 & 0.93 & 3.24 & 0.46 \\
\hline le & 18.87 & 0.00 & 6.60 & 7.08 & 0.00 & 0.00 & 18.87 & 6.13 & 36.79 & 1.42 & 2.36 & 1.88 \\
\hline ld & 9.91 & 0.00 & 7.08 & 6.13 & 0.00 & 0.47 & 23.58 & 6.13 & 36.32 & 2.36 & 1.42 & 6.60 \\
\hline le & 18.06 & 0.46 & 10.65 & 17.13 & 0.00 & 0.00 & 21.76 & 6.94 & 12.04 & 0.00 & 0.46 & 12.50 \\
\hline $2 \mathrm{a}$ & 21.78 & 0.00 & 5.94 & 31.68 & 2.48 & 0.49 & 7.92 & 0.99 & 27.72 & 0.49 & 0.49 & 0.00 \\
\hline $2 b$ & 36.95 & 0.00 & 0.00 & 50.74 & 5.91 & 0.00 & 0.49 & 0.49 & 5.42 & 0.00 & 0.00 & 0.00 \\
\hline $3 \mathrm{a}$ & 7.32 & 0.00 & 4.39 & 22.93 & 2.43 & 0.00 & 3.90 & 0.49 & 57.56 & 0.98 & 0.00 & 0.00 \\
\hline $3 b$ & 40.60 & 0.00 & 0.43 & 42.74 & 4.70 & 0.00 & 2.13 & 0.00 & 8.55 & 0.00 & 0.00 & 0.85 \\
\hline $4 a$ & 52.15 & 0.00 & 0.48 & 28.23 & 3.34 & 0.00 & 7.18 & 0.96 & 7.66 & 0.00 & 0.00 & 0.00 \\
\hline $4 b$ & 14.29 & 0.00 & 13.85 & 24.24 & 0.87 & 0.00 & 7.79 & 4.76 & 29.87 & 2.16 & 2.16 & 0.00 \\
\hline $4 c$ & 35.48 & 0.00 & 0.92 & 50.69 & 5.07 & 0.00 & 0.46 & 0.00 & 7.37 & 0.00 & 0.00 & 0.00 \\
\hline $5 \mathrm{a}$ & 35.19 & 0.00 & 4.17 & 33.79 & 1.85 & 0.00 & 5.09 & 0.93 & 18.06 & 0.46 & 0.00 & 0.46 \\
\hline $5 b$ & 8.69 & 0.00 & 3.86 & 3.86 & 0.97 & 0.00 & 13.53 & 4.35 & 61.35 & 1.93 & 1.45 & 0.00 \\
\hline $5 \mathrm{c}$ & 40.47 & 0.00 & 0.00 & 43.98 & 3.70 & 0.00 & 3.70 & 0.93 & 6.94 & 0.00 & 0.00 & 0.00 \\
\hline $6 a$ & 30.29 & 0.00 & 1.44 & 39.42 & 2.88 & 0.00 & 5.77 & 0.48 & 18.75 & 0.96 & 0.00 & 0.00 \\
\hline $6 \mathrm{~b}$ & 36.91 & 0.00 & 0.47 & 43.92 & 2.34 & 0.00 & 3.74 & 0.47 & 11.68 & 0.47 & 0.00 & 0.00 \\
\hline $7 \mathrm{a}$ & 3.45 & 0.00 & 0.99 & 5.42 & 0.00 & 0.00 & 19.70 & 3.94 & 59.61 & 1.48 & 0.00 & 5.41 \\
\hline $7 \mathrm{~b}$ & 43.93 & 0.00 & 2.80 & 25.70 & 2.34 & 0.00 & 2.80 & 0.00 & 15.42 & 0.00 & 0.00 & 7.01 \\
\hline $8 a$ & 43.22 & 0.00 & 0.50 & 40.70 & 0.00 & 0.00 & 4.02 & 0.00 & 9.05 & 0.00 & 0.00 & 2.51 \\
\hline $8 b$ & 8.92 & 0.00 & 5.63 & 2.35 & 0.00 & 0.00 & 13.62 & 5.63 & 58.22 & 5.16 & 0.47 & 0.00 \\
\hline $8 c$ & 18.78 & 0.00 & 13.15 & 13.15 & 0.00 & 0.47 & 12.21 & 4.23 & 36.15 & 0.94 & 0.46 & 0.46 \\
\hline $8 d$ & 16.20 & 0.46 & 14.81 & 8.33 & 0.46 & 1.39 & 13.43 & 4.17 & 33.33 & 7.41 & 0.00 & 0.00 \\
\hline $9 \mathrm{a}$ & 7.76 & 0.00 & 26.48 & 0.00 & 0.00 & 0.46 & 29.68 & 9.13 & 22.37 & 4.11 & 0.00 & 0.00 \\
\hline $9 \mathrm{~b}$ & 21.52 & 0.00 & 11.66 & 2.70 & 0.00 & 0.45 & 27.35 & 6.28 & 25.56 & 4.48 & 0.00 & 0.00 \\
\hline 9 & 26.89 & 0.00 & 8.02 & 13.21 & 0.00 & 3.77 & 16.98 & 2.36 & 25.47 & 3.30 & 0.00 & 0.00 \\
\hline $9 \mathrm{~d}$ & 54.41 & 0.00 & 0.47 & 24.65 & 0.00 & 0.00 & 11.16 & 0.47 & 8.37 & 0.00 & 0.47 & 0.00 \\
\hline $10 \mathrm{a}$ & 48.33 & 0.00 & 0.00 & 33.96 & 0.00 & 0.00 & 7.18 & 0.48 & 9.57 & 0.00 & 0.00 & 0.48 \\
\hline $10 \mathrm{~b}$ & 44.55 & 0.00 & 1.42 & 36.97 & 0.95 & 0.00 & 4.74 & 0.00 & 9.95 & 0.00 & 0.00 & 1.42 \\
\hline $11 \mathrm{a}$ & 41.83 & 0.00 & 1.92 & 14.42 & 0.00 & 0.48 & 13.46 & 0.00 & 26.92 & 0.96 & 0.00 & 0.00 \\
\hline $11 \mathrm{~b}$ & 51.67 & 0.00 & 0.48 & 22.49 & 0.00 & 0.00 & 12.92 & 0.00 & 11.96 & 0.00 & 0.00 & 0.48 \\
\hline $12 \mathrm{a}$ & 45.70 & 0.00 & 5.60 & 29.00 & 0.90 & 0.30 & 4.70 & 0.30 & 11.70 & 0.30 & 0.00 & 1.50 \\
\hline $12 \mathrm{~b}$ & 35.91 & 0.00 & 3.01 & 21.26 & 0.60 & 0.30 & 14.54 & 3.41 & 16.75 & 0.30 & 0.30 & 3.91 \\
\hline
\end{tabular}


Table 5. Environment determination and $\operatorname{Rr}(\%)$ values

\begin{tabular}{|c|c|c|c|c|c|c|c|c|c|c|c|}
\hline Sample & TPI & GI & $\mathrm{A} \%$ & $\mathrm{~B} \%$ & $\mathrm{C} \%$ & $\mathrm{D} \%$ & $\mathrm{~A}+\mathrm{D} \%$ & $\begin{array}{l}\text { Dep. Envir. } \\
\text { (An. Mac.) }\end{array}$ & $\begin{array}{l}\text { Dep. Envir. } \\
\text { (Microlit.) }\end{array}$ & Conclusion & $\operatorname{Rr}(\%)$ \\
\hline $1 \mathrm{a}$ & 4.53 & 198.00 & 8.49 & 0.00 & 78.77 & 12.74 & 21.23 & Wet Forest & $\begin{array}{l}\text { Limnic to Swamp } \\
\text { Forest }\end{array}$ & SWAMP FOREST & 0.41 \\
\hline $1 b$ & 32.87 & 4.19 & 32.87 & 9.72 & 55.56 & 1.85 & 34.72 & Swamp Forest & Swamp Forest & SWAMP FOREST & 0.50 \\
\hline $1 \mathrm{c}$ & 0.39 & 3.00 & 39.15 & 12.74 & 44.81 & 3.30 & 42.45 & Reed Moor & Reed Moor & REED MOOR & 0.56 \\
\hline $1 d$ & 0.29 & 2.67 & 37.74 & 13.21 & 39.62 & 9.43 & 47.17 & Reed Moor & Reed Moor & REED MOOR & 0.55 \\
\hline $1 \mathrm{e}$ & 0.48 & 2.08 & 12.50 & 17.59 & 56.94 & 12.50 & 25.00 & Reed Moor & Wet Forest & WET FOREST & 0.54 \\
\hline $2 \mathrm{a}$ & 0.59 & 5.81 & 30.69 & 6.93 & 61.39 & 0.99 & 31.68 & Limnic & $\begin{array}{l}\text { Limnic to Swamp } \\
\text { Forest }\end{array}$ & LIMNIC & 0.41 \\
\hline $2 b$ & 0.79 & 173.00 & 11.33 & 0.49 & 88.18 & 0.00 & 11.33 & $\begin{array}{l}\text { Wet Forest to } \\
\text { Swamp Forest }\end{array}$ & $\begin{array}{l}\text { Wet Forest to } \\
\text { Swamp Forest }\end{array}$ & $\begin{array}{l}\text { WET FOREST TO } \\
\text { SWAMP FOREST }\end{array}$ & 0.42 \\
\hline $3 a$ & 0.17 & 3.57 & 60.00 & 4.88 & 34.15 & 0.97 & 60.97 & Reed Moor & Reed Moor & REED MOOR & 0.36 \\
\hline $3 b$ & 1.04 & 32.00 & 13.25 & 0.43 & 85.47 & 0.85 & 14.10 & Swamp Forest & Swamp Forest & SWAMP FOREST & 0.43 \\
\hline $4 a$ & 1.57 & 37.00 & 11.00 & 1.44 & 87.56 & 0.00 & 11.00 & Swamp Forest & $\begin{array}{l}\text { Swamp Forest to } \\
\text { Wet Forest }\end{array}$ & SWAMP FOREST & 0.33 \\
\hline $4 b$ & 0.77 & 2.24 & 32.90 & 18.61 & 46.32 & 2.16 & 35.06 & Reed Moor & $\begin{array}{l}\text { Wet Forest to } \\
\text { Reed Moor }\end{array}$ & REED MOOR & 0.40 \\
\hline $4 \mathrm{c}$ & 0.85 & 42.50 & 12.44 & 0.92 & 86.64 & 0.00 & 12.44 & $\begin{array}{l}\text { Swamp Forest } \\
\text { (Limnic) }\end{array}$ & Swamp Forest & SWAMP FOREST & 0.41 \\
\hline $5 \mathrm{a}$ & 0.95 & 11.06 & 19.91 & 5.09 & 74.07 & 0.93 & 20.84 & Limnic & $\begin{array}{l}\text { Limnic to Swamp } \\
\text { Forest }\end{array}$ & LIMNIC & 0.43 \\
\hline $5 b$ & 0.24 & 2.11 & 63.77 & 8.21 & 26.09 & 1.93 & 65.70 & Reed Moor & Reed Moor & REED MOOR & 0.28 \\
\hline $5 \mathrm{c}$ & 1.12 & 29.33 & 10.65 & 0.93 & 88.42 & 0.00 & 10.65 & $\begin{array}{l}\text { Swamp Forest } \\
\text { (Limnic) }\end{array}$ & $\begin{array}{l}\text { Swamp Forest to } \\
\text { Wet Forest }\end{array}$ & SWAMP FOREST & 0.38 \\
\hline $6 \mathrm{a}$ & 0.62 & 19.00 & 21.63 & 1.92 & 75.48 & 0.96 & 22.59 & Limnic & Limnic & LIMNIC & 0.40 \\
\hline $6 \mathrm{~b}$ & 1.00 & 27.00 & 14.02 & 0.93 & 84.58 & 0.47 & 14.49 & $\begin{array}{l}\text { Swamp Forest } \\
\text { (Limnic) }\end{array}$ & Swamp Forest & SWAMP FOREST & 0.47 \\
\hline $7 \mathrm{a}$ & 0.15 & 2.81 & 59.61 & 4.93 & 28.57 & 6.90 & 66.51 & Reed Moor & Reed Moor & REED MOOR & 0.40 \\
\hline $7 b$ & 1.22 & 21.22 & 17.76 & 2.80 & 72.43 & 7.01 & 24.77 & Limnic & Limnic & LIMNIC & 0.43 \\
\hline $8 \mathrm{a}$ & 0.89 & 46.00 & 9.05 & 0.50 & 87.94 & 2.51 & 11.56 & Swamp Forest & Swamp Forest & SWAMP FOREST & 0.41 \\
\hline $8 b$ & 0.34 & 2.27 & 58.69 & 11.27 & 24.88 & 5.16 & 63.85 & Reed Moor & Reed Moor & REED MOOR & 0.35 \\
\hline $8 \mathrm{c}$ & 0.71 & 2.67 & 36.62 & 17.37 & 44.13 & 1.88 & 38.50 & Reed Moor & $\begin{array}{l}\text { Reed Moor to } \\
\text { Wet Forest }\end{array}$ & REED MOOR & 0.57 \\
\hline $8 \mathrm{~d}$ & 0.68 & 1.74 & 33.79 & 18.98 & 37.96 & 8.80 & 42.59 & Reed Moor & Reed Moor & REED MOOR & 0.57 \\
\hline $9 \mathrm{a}$ & 0.70 & 1.01 & 22.37 & 35.62 & 37.44 & 4.57 & 26.94 & Dry Forest & Dry Forest & DRY FOREST & 0.47 \\
\hline $9 b$ & 0.70 & 2.32 & 25.56 & 17.94 & 51.57 & 4.93 & 30.49 & Reed Moor & Wet Forest & $\begin{array}{l}\text { WET FOREST TO } \\
\text { REED MOOR }\end{array}$ & 0.51 \\
\hline $9 \mathrm{c}$ & 1.36 & 3.56 & 25.47 & 10.38 & 57.07 & 7.08 & 32.55 & Wc & Wet Fo & WET FOREST & 0.49 \\
\hline $9 \mathrm{~d}$ & 2.04 & 28.57 & 8.84 & 0.93 & 90.23 & 0.00 & 8.84 & Swamp Forest & $\begin{array}{l}\text { Wet Forest to } \\
\text { Swamp Forest }\end{array}$ & $\begin{array}{l}\text { SWAMP FOREST } \\
\text { TO WET FOREST }\end{array}$ & 0.43 \\
\hline $10 \mathrm{a}$ & 1.07 & 28.00 & 9.57 & 0.48 & 89.47 & 0.48 & 10.05 & Swamp Forest & $\begin{array}{l}\text { Wet Forest to } \\
\text { Reed Moor }\end{array}$ & SWAMP FOREST & - \\
\hline $10 \mathrm{~b}$ & 1.24 & 27.57 & 10.90 & 1.42 & 86.26 & 1.42 & 12.32 & Swamp Forest & Swamp Forest & SWAMP FOREST & \\
\hline $11 \mathrm{a}$ & 0.93 & 12.40 & 26.92 & 1.92 & 69.71 & 1.44 & 28.36 & Limnic & Limnic & LIMNIC & $\overline{0.41}$ \\
\hline $11 \mathrm{~b}$ & 1.31 & 27.43 & 11.96 & 0.48 & 87.08 & 0.48 & 12.44 & Swamp Forest & Swamp Forest & SWAMP FOREST & 0.37 \\
\hline $12 \mathrm{a}$ & 0.75 & 8.99 & 0.00 & 5.91 & 79.48 & 0.00 & 14.61 & Limnic & Swamp Forest & SWAMP FOREST & 0.49 \\
\hline $12 b$ & 1.07 & 5.27 & 0.00 & 6.42 & 71.72 & 0.00 & 21.87 & $\begin{array}{l}\text { Limnic to Swamp } \\
\text { Forest }\end{array}$ & $\begin{array}{l}\text { Limnic to Swamp } \\
\text { Forest }\end{array}$ & $\begin{array}{l}\text { LIMNIC TO SWAMP } \\
\text { FOREST }\end{array}$ & 0.50 \\
\hline
\end{tabular}

The microlithotype results indicate a dominant $C$ parameter, initially evidence of a continuously wet forest swamp with high water tables (Diessel, 1992). Apex $A+D$ is relatively well represented, which suggests that the peat was periodically invaded by herbaceous vegetation. The $B$ zone is poorly expressed, which excludes the influence of terrestrial environments. The $D$ parameter, which represents lakes that collected detritus from other environments, is the least represented zone. Generally, the coal sample distribution shows a trend from forest environments with high water tables to herbaceous environments with variable water tables.

The vitrinite reflectance values (Table 5) vary from $0.28 \%$ to $0.57 \%$, with a mean value of $0.44 \%$, reflecting relatively immature organic matter. In the Menefee Formation, for a given outcrop with no apparent thermal disturbance, the sample ranks do not vary. Effectively, this parameter does not exhibit a monotonic trend from the base to the top of the Menefee 
Formation, but reveals, instead, much more complex fluctuations (Buillit, 2000). As previously mentioned, these results imply that the measured variations can be linked either to coal composition or to redox fluctuations during the deposition (no evidence of recent oxidation has been observed in the samples). Measurements of $\operatorname{Rr} \%$ were always performed on a given petrographic constituent, i.e. homogenous, non-microporous, and non-fluorescent telocollinite particles of regular shape and with no inclusions. These precautions strongly limit the influence of the coal composition, i.e. the nature of the precursor, within $\operatorname{Rr} \%$ variations. Moreover, we performed micro-infrared studies in two samples from the Farmington seam, 9b and 9d. Sample 9b corresponds to a wet forest/reed moor environment with a $\mathrm{Rr}$ value of $0.51 \%$ whereas sample $9 \mathrm{~d}$ corresponds to a swamp forest/wet forest environment with a $\mathrm{Rr}$ of $0.41 \%$. As shown in Fig. $6 a$, the $\mathrm{OH}$ band and the $\mathrm{C}=\mathrm{O} / \mathrm{C}=\mathrm{C}$ ratio are higher for sample $9 \mathrm{~b}$, clearly indicating the influence of oxidation process. Compared to a trend of normal coal in a micro-infrared parameter versus Rr\% crossplot (Fig. 6b), a correction can be applied to the $\mathrm{Rr} \%$ to estimate its real rank value (about $0.40 \% \mathrm{Rr}$ ). In the Farmington seam, $\mathrm{Rr} \%$ variations will be interpreted in terms of redox processes on a given precursor during deposition. 


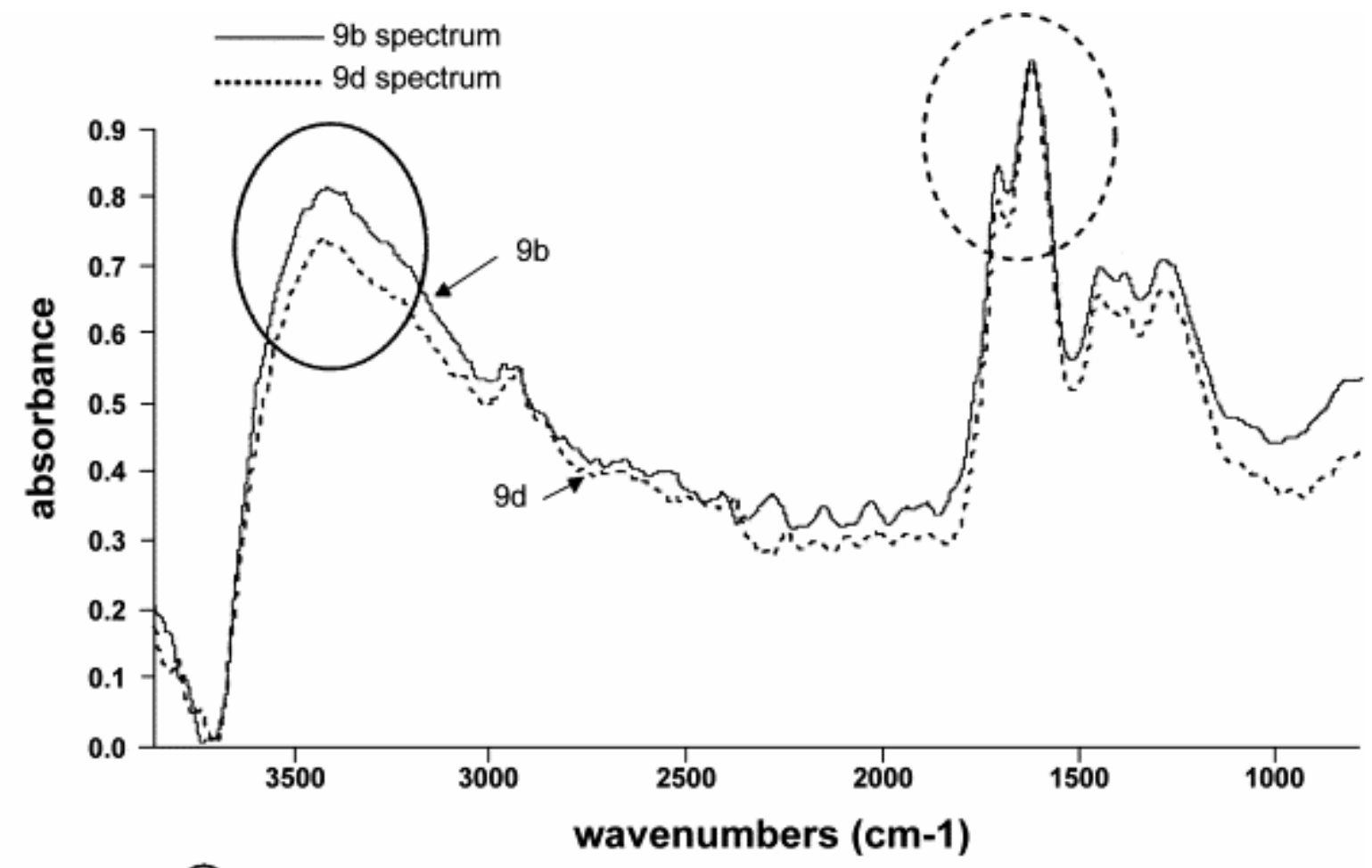

(a) $\because, \mathrm{C}=\mathrm{O} / \mathrm{C}=\mathrm{C}$ ratio higher for $9 \mathrm{~b}$

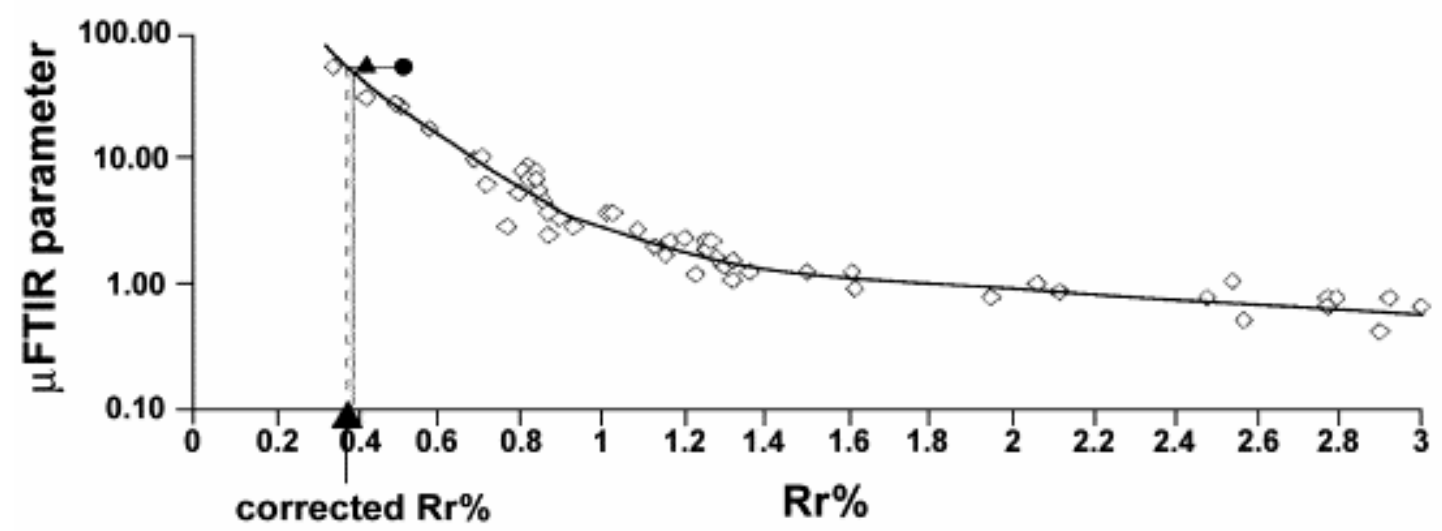

coals - reference samples

(b) $\quad \therefore 9 \mathrm{~d}$

Fig. 6. Micro-infrared analyses of two coals from the same seam: (a) micro-infrared spectra, and (b) equivalent Rr\% corrected by micro-infrared analyses. 


\section{Discussion}

\subsection{Advantages and signification of the Nicolas et al. (1998) model}

After comparing the Diessel and Hacquebard-Donaldson/Marchioni diagrams, Nicolas et al. (1998) proposed a model for littoral domains, suggesting that the eight reconstructed environments are distributed in a logical order from continental to marine (Fig. 7a): terrestrial, dry forest, wet forest, swamp forest, limnic, reed moor, swamp, and limnic-lagunar. In this study, we assigned numbers for the palaeoenvironments, from 1 for terrestrial, to 8 for the limnic-lagunar. This palaeogeographic continuum accounts for fluctuations in the water table, whether due to variations in sea level variation or in a subsidence rate. However, the location of limnic and reed moor environments within the model must be taken with caution. Firstly, limnic environments might be present in a number of other locations than the one illustrated, as lakes can form in any local depression. Concerning the Mesa Verde littoral, the very minor differences (Navarre, 1995) makes it highly unlikely that lakes would have formed in such depressions. Secondly, reed moor can represent either an extensive marshy environment, or a limited reed belt that developed around a lake (e.g., the Annecy Lake- Buillit et al., 1997). However, owing to the contact between both environments in the model, the only criterion to be taken into consideration in the reconstruction is the extent of reed moor environment.

A comparison of the environmental positions in Nicolas et al. (1998) model to the mean reflectance value was tested on a crossplot diagram (Fig. 8). The diagram shows a generally negative trend, excluding four reed moor environments represented by the samples 1c, 1d, 8c, and 8d (if the Reflectance values are taken into account, the location of these environments would be close to dry forest and wet forest). As attested by micro-infrared studies, Rr\% fluctuations mainly express the degree of vitrinite oxidation. In this case, the negative relationship is consistent with the palaeogeographic reconstruction proposed by Nicolas et al. (1998). Effectively, the more continental environments are preserved under variable water table levels leading to recurrent aerial exposure of the peat constituents, and high $\mathrm{Rr} \%$ values.

Oppositely, the more littoral environments are preserved under constantly high water table levels, due to the proximity of the sea, and the peat constituents are rarely exposed to the air. The Rr\% values are lower. The Nicolas et al. (1998) distribution can then be considered as a reliable palaeogeographic continuum, accounting for variable humidity conditions within the considered environments. Nevertheless, no relevant argument can demonstrate the origin of these humidity variations, i.e. climatic or eustatic. 


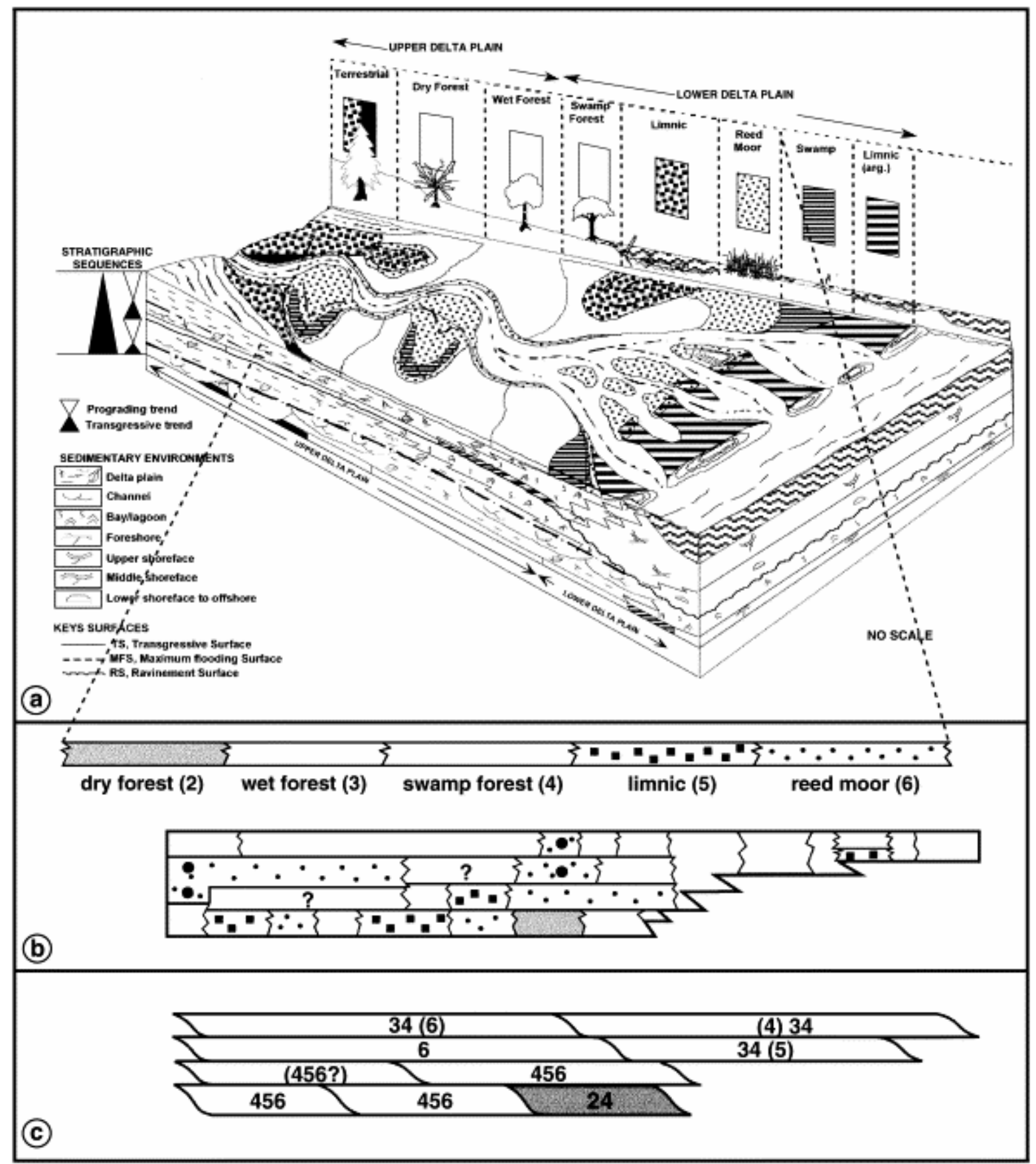

Fig. 7. (a) Ideal depositional environments of deltaic coal beds to the sedimentological facies in a fluvio-deltaic domain (Nicolas et al., 1998), (b) distribution of the environments in the studied coal seam, and (c) the nine palaeogeographic sequences identified within the Farmington coal. 


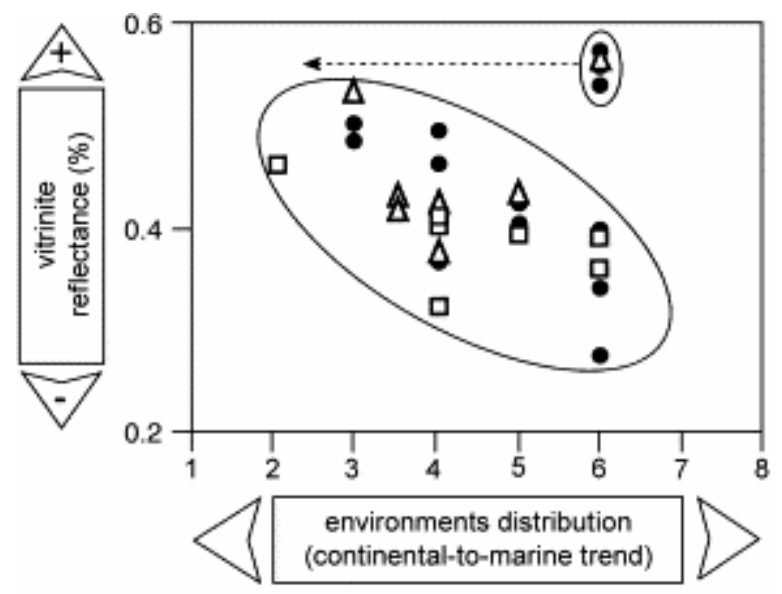

$\Delta$ top of the seam

- middle of the seam

a bottom of the seam

Fig. 8. Illustration of the relationship between the environments distribution and the vitrinite reflectance. The dashed line arrow indicates the expected location for the four environments that does not fit with the trend.

\subsection{Distribution of the environments within the Farmington coal. Comparison with the Nicolas et al. (1998) model}

The environments deduced from the petrographic analyses of the 33 samples were replaced in the schematic coal layer (Fig. 7b). First of all, the four reed moor environments which do not fit into the crossplot in Fig. 8, represented by black dots in Fig. 7b, are all close to wet forest environments, which is to be expected considering their degree of oxidation. They can be interpreted as transitional environments between reed moor and wet forest, probably temporary dry. Secondly, the reconstruction reveals a regular pattern within the seam. The latter can be divided into nine sequences consisting in short palaeogeographic successions of the Nicolas et al. (1998) model. Taking into account the numbers assigned to each environment, 1 to 8, different kinds of successions can be distinguished (Fig. 7c): one "2-4" succession, three "3-4" successions, (3) three to four "4-5-6" successions, and (4) one "6" succession. The following question arises: do these successions really represent separated sequences or do they only express, for a given horizontal level, lateral diversity of timeequivalent units? To answer that question, one can consider similar present-day environments, such as the Florida Everglades, the Okefenokee Swamps in Georgia, or the Guadeloupe Mangrove (e.g., Lallier-Vergès et al., 1998). These environments require such stable conditions to develop that their regular offshore to onshore distribution seldom allows lateral variations. A so constantly repetitive distribution within the Farmington seam could hardly be explained by palaeogeographic factors. Such successions more likely suggest the record of a landscape migration, well known for present-day mangrove littorals (e.g., Lallier-Vergès et al., 1998).

\subsection{Sequence stratigraphy implications}

The features observed in coal seams in littoral domains have been linked to the sequence stratigraphy settings by several authors (e.g., Cross; Diessel and Bohacs). Indeed, coal characteristics in such domains depend on the balance between accommodation $(A)$, peat production rate (PPR), and sedimentary supply $(S)$. Bohacs and Suter (1997) have discussed 
the importance of the A/PPR ratio in the preservation of peat as coal. When $\mathrm{A}$ is low, peat produces in situ organic matter until the available space is used up. Then, it can either accumulate laterally, or stop accumulating: coal layers are generally broad and relatively thick. If $A$ compensates PPR, vertical accumulation can occur whereas if $A$ exceeds PPR, peatlands are flooded, and their organic matter is dispersed in the basin created within siliciclastic sediments. For these high $A$ rates, two ratios must be taken account: $A / \mathrm{PPR}$ and $A / S$.

If the Bohacs and Suter (1997) model concerns the distribution of successive coal seam in a cycle of accommodation change, one can use these results for fluctuations at a higher scale. Herein, we propose to apply these concepts to explain the landscape migration observed within the 9 sequences of the Farmington coal seam. The interpretation is illustrated in Fig. 9:

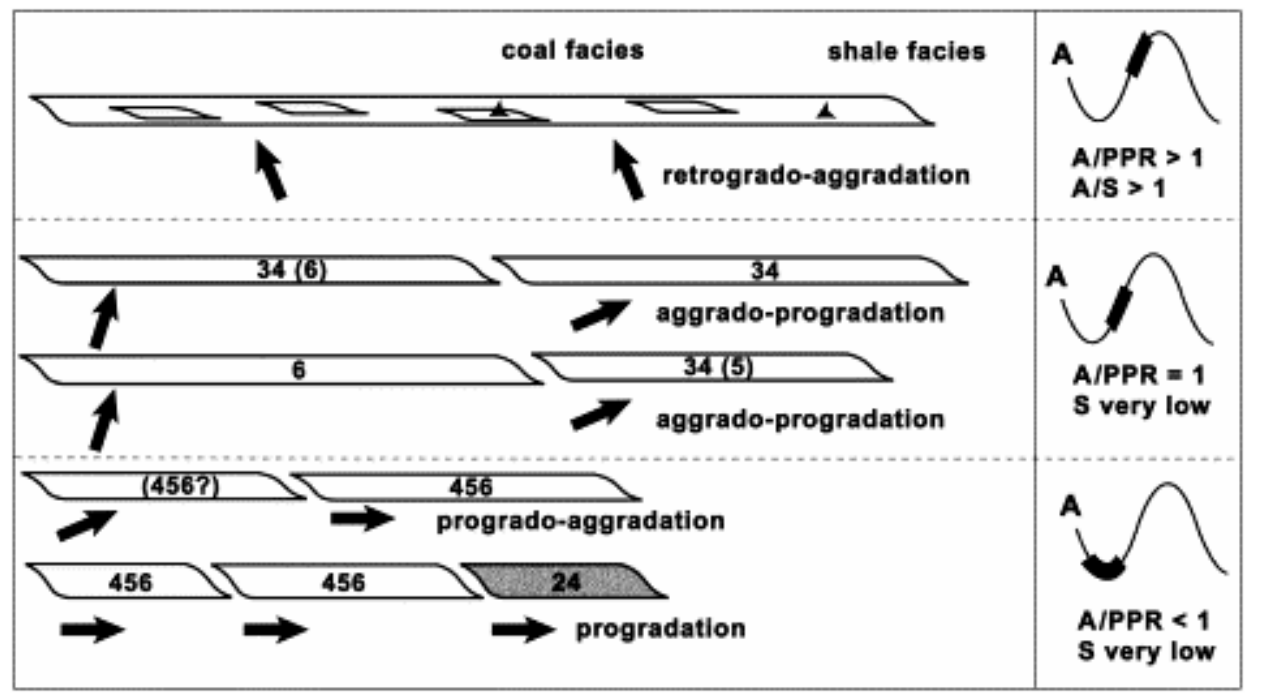

Fig. 9. Schematic model for explaining the distribution of the nine sequences within the Farmington coal. Relationships with the balance between accommodation $(A)$, peat production rate (PPR), and sedimentary supply $(S)$.

(1) The first five sequences defined in the seam consist essentially of the same "4-5-6" succession. These successions are short and highly repetitive, so that their distribution clearly suggests little available space for the peat to extend. As these five first sequences were preserved under low $A$ values, progradation occurred due to a progressive lateral migration of the peat. Landscapes shifted progressively seaward. The "2-4" sequence preserved at the bottom right of the seam probably recorded a remaining local positive relief in the initial landscape.

(2) The last four coal sequences consist of "3-4" and "6" sequences. These successions are more extensive, more homogenous, and less repetitive than the first five. Such features certainly indicate an increase in the available space, as a given landscape can accumulate both vertically and laterally. The more humid sequence composed of reed moor environment (number 6), probably developed first with no spatial limitation as an aggradational succession. Then, as peat production compensated the space created, this spatial limitation led to the setting-in of less humid environments, i.e. wet forest and swamp forest that shifted towards a more progradational trend. 
Although this succession is interpreted as a progradational-aggradational architecture, the implication of the autocyclic factors can also be invoked, Firstly, the high diversity of the environments at the base of the seam is probably amplified by the topographic differences inherited from the initial surface. Secondly, the global change from herbaceous environments at the bottom of the seam to forest environments at its top may also indicate warming-up of the climate during the 5000- to 10,000-year period corresponding to the 80 -cm thickness of the coal. Nevertheless, all the interpretations proposed herein are based on the balance between accommodation, peat production rate and sedimentary supply. No direct relationship with either eustatic or climatic changes can be proved.

\subsection{Towards the definition of genetic units in the Mesa Verde Group?}

As coal-shale doublets developed within all the aggradational grouped genetic units of the deltaic-plain coal and shale are most probably genetically linked within each doublet. To corroborate this, a study was conducted on the organic content of various shales comprised in the uppermost part of the coals. The palynofacies composition (Buillit, 2000) revealed a dominant terrestrial contribution, identified under incident light as essentially composed of vitrinite particles from peatland environments. Two features of this maceral-like analysis were also significant: (1) the reflectance values of the vitrinite vary from $0.22 \%$ to $0.48 \%$, with a mean value around $0.36 \%$, (2) inertinite macerals are practically nonexistent. As mean $\mathrm{Rr} \%$ values from the shale vitrinite are lower than mean $\mathrm{Rr} \%$ values than the coal vitrinites (microinfrared corrected at $0.40 \%$ ), oxidation process on dispersed organic matter must have been limited. These two results suggest that the organic matter scattered in the shale facies derives from the flooding of adjacent peatlands (Fig. 9). Then, the coal-shale doublets exhibit a progressive rise in the water table, which contributed to: (1) in situ accumulation and progradation of vegetal debris, (2) in situ accumulation and aggradation of vegetal debris, and (3) dispersal of vegetal debris within trapped flooding sediments. This succession can be interpreted as the elemental cycle of Base Level changes in the deltaic-plain of the Menefee Formation. It consists of a progradational-aggradational retrogradational genetic units. Considering the repetition of such doublets over the entire deltaic-plain of the Menefee Formation, the existence of genetic units in this part of the continental deposits can be generalised to the entire long-term cycle. This is illustrated in Fig. 10.

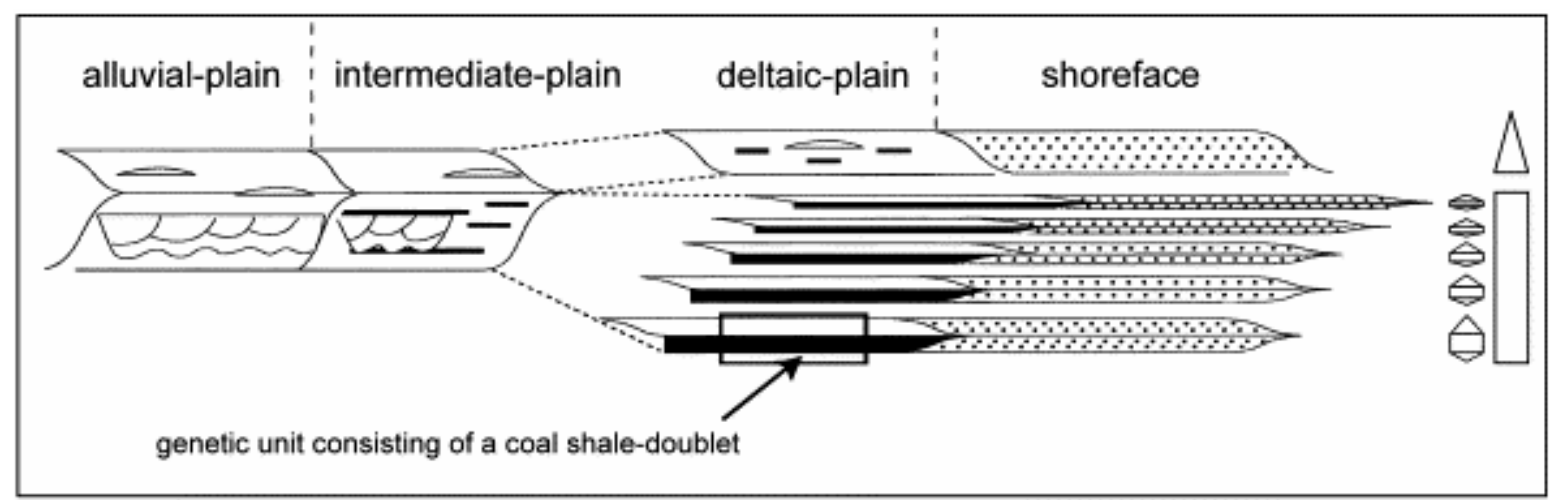

Fig. 10. The stacked genetic units in the deltaic-plain domain. 


\section{Summary and conclusions}

The deltaic-plain facies of the Mesa Verde Group offer opportunities to study littoral coal seams and their meaning in terms of sequence stratigraphy. The distribution of these coals within the entire 3-4 Ma regressive-transgressive cycle previously led to the definition of grouped genetic units. The present high-resolution petrographic study conducted on 33 samples taken from an 80 -cm thick, $1.5-\mathrm{km}$ long coal seam and its uppermost shales in Farmington outcrop has delivered new results.

The reconstruction of the environments resulting from the petrographic studies reveal the predominance of two groups, i.e. herbaceous environments represented by limnic and reed moor environments and forest environments represented by swamp forest and wet forest environments. A relationship has been observed between the environments and the vitrinite oxidation (the higher the degree of humidity the lower the Rr\%) attesting the validity of the palaeogeographic distribution of these environments obtained by the model proposed by Nicolas et al. (1998) for such domains. In the Farmington coal seams, the distribution of these environments reveals the existence of nine sequences. Taking into account the palaeogeographic location of the environments within the model, as well as the extent of the sequences, this distribution is interpreted in terms of the balance between accommodation, peat production rate, and sedimentary supply. The study on the uppermost shales revealed that their organic matter derived from the flooding of adjacent peats. The coal-shale doublets is therefore interpreted as a complete cycle of Base Level change, i.e. an elemental progradational-aggradational-retrogradational genetic unit of the deltaic-plain of the Menefee Formation. Its definition is extended to all the deltaic-plain coals that are subsequently the only facies of the Menefee formation that have recorded the prograding events.

This interpretation conveys, for the first time, Walther's law (Walther, 1893-1894) for coal seams vertical successions of facies correspond to the record of landscape migrations Indeed even though Walter's law is often used to define sedimentary sequences, coals are usually considered by sedimentologists as one-off events. Therefore, precautions must be taken concerning space-time correlations based on coal beds, and Sequence Stratigraphy concepts should stress the fact that coals are not true isochronous surfaces. In coals, time is not recorded linearly, but rather diagonally: many episodes can be recorded within a given horizontal trend.

\section{Acknowledgements}

This work was conducted with the financial support of TotalFinaElf. The fieldwork in the Indian reservations was made possible thanks to the local support of Ute and Navajo tribes. We would also like to thank Marielle Hatton for technical assistance.

Special thanks are extended to François Baudin, Remi Eschard, and François Guillocheau for fruitful discussions.

\section{References}

Bohacs and Suter, 1997. K. Bohacs and J. Suter, Sequence stratigraphic distribution of coaly rocks: fundamental controls and paralic examples. AAPG Bull. 81 (1997), pp. 1612-1639. 
Buillit, 2000. Buillit, N., 2000. Marqueurs Organiques et Stratigraphie Génétique - Le prisme fluvio-deltaïque campanien du groupe du Mesa Verde (Colorado, EU). Thése de l'Université d'Orléans, 335 pp.

Buillit et al., 1997. N. Buillit, E. Lallier-Vergés, J.R. Disnar and J.L. Loizeau , Changements climatiques et effets anthropiques au cours du dernier millénaire attestés par l'étude pétrographique de la matiére organique (Annecy, Le Petit Lac; France). Bull. Soc. Géol. Fr. 168 (1997), pp. 573-583.

Cobban et al., 1994. W.A. Cobban, E.A. Merewether, T.D. Fouch and J.D. Obradovich , Some Cretaceous shorelines in the western interior of the United States. In: M.V. Caputo, J.A. Peterson and K.J. Franczyk, Editors, Mesozoic Systems of Rocky Mountain Region, USA (1994), pp. 393-413.

Cross, 1986. T.A. Cross , Tectonic controls of foreland basin subsidence and Laramide style deformation, western United State. In: P.A. Allen and P. Homewood, Editors, Foreland BasinsInt. Assoc. Sedimentol. Spec. Publ. vol. 8, Blackwell Scientific Publications, Oxford (1986), pp. 15-39.

Cross, 1988. T.A. Cross , Controls on coal distribution in transgressive-regressive cycles, Upper Cretaceous, Western Interior, U.S.A. Soc. Econ. Paleontol. Mineral. (1988), pp. 371380.

Diessel, 1986. C.F.K. Diessel , On the correlation between coal facies and depositional environments. Advances in the study of the Sydney basin. 20 Newcastle Symp. Proc. 246 (1986), pp. 19-22.

Diessel, 1992. C.F.K. Diessel , Coal-Bearing Depositional Systems. , Springer-Verlag, Berlin (1992) 721 pp. .

Eschard et al., 1994. R. EschardHersesim group and G. Desaubliaux , Sequence stratigraphy of a major regressive-transgressive littoral clastic wedge in the Mesa Verde are (Campanian, Colorado). In: Field Trip Guide Book IFP, ref. IFP 41241, IFP, Reuil-Malmaison (1994).

Gentzis et al., 1992. T. Gentzis, F. Goodarzi and R.A. McFarlane , Molecular structure of reactive coals during oxidation, carbonization and hydrogenation. An infrared photoacoustic spectroscopic and optical microscopy study. Org. Geochem. 18 (1992), pp. 249-258.

Hacquebard and Donaldson, 1969. P.A. Hacquebard and J.R. Donaldson , Carboniferous coal deposition associated with flood-plain and limnic environments in Nova Scotia. In: E.C. Dapples and M.E. Hopkins, Editors, Environments of Coal DepositionGeol. Soc. Am. Spec. Pap. vol. 114 (1969), pp. 143-191.

Homewood et al., 1992. P. Homewood, F. Guillocheau, R. Eschard and T.A. Cross , High resolution correlations and genetic stratigraphy: an integrated approach. BCREDP 16 (1992), pp. 375-381.

International Committee for Coal Petrology, 1963-1993. International Committee for Coal Petrology, 1963-1993. International Handbook of Coal Petrology, 2nd ed., CNRS ed., Paris, 
184 pp.; repr. 1985; 1st supp., 1971, 197 pp., repr. 1985; 2nd suppl., 1975, 60 pp.; 3rd suppl., 1993, 88 pp.

Kauffman, 1977. E.G. Kauffman , Geological and biological overview: Western Interior Cretaceous Basin. Mt. Geol. 14 (1977), pp. 75-99.

Lallier-Vergès et al., 1998. E. Lallier-Vergès, B.P. Perrussel, J.R. Disnar and F. Baltzer , Relationships between environmental conditions and the diagenetic evolution of organic matter derived from higher plant in a modern mangrove swamp system (Guadeloupe, French West Indies). Org. Geochem. 29 (1998), pp. 1663-1686.

Marchioni, 1980. D.L. Marchioni , Petrography and depositional environment of the Liddell Seam, Upper Hunter Valley, New South Wales. Int. J. Coal Geol. 1 (1980), pp. 35-61.

Molenaar and Baird, 1992. C.M. Molenaar and J.K. Baird , Regional stratigraphic cross sections of Upper Cretaceous rocks across the San Juan Basin, northwestern New Mexico and southern Colorado. U. S. Geol. Surv. Open-File Rep. 92-257 (1992) 3 sheets .

Navarre, 1995. Navarre, J.C., 1995. Stratigraphie génétique du Mesa Verde (Basin de San Juan); Préservation différentielle des environnements de plaine côtière et de littoral dominé par les vagues. Thèse de l'université de Rennes I, 219 pp.

Nicolas et al., 1998. G. Nicolas, B. Pradier and F. Vannier-Petit, Reconstitution des environnements de dépôt des sédiments organiques de plaine deltaïque. Application à l'étude sédimentologique du groupe du Brent (mer du Nord). Bull. Cent. Rech. Explor. Prod. ElfAquitaine, Publ. Spec. 21 (1998), pp. 249-264.

Powell, 1897. J.W. Powell , Exploration of the Colorado River of the West and its tributaries. , Smithsonian Institute, Washington, DC (1875) 291 pp. .

Ruau et al., 1995. O. Ruau, B. Pradier, P. Landais and J.L. Gardette , Influence of the condition of the deposition on the chemistry and the reflectance variation of the Brent coals. Org. Geochem. (1995), pp. 325-339.

Seyler, 1931. C.A. Seyler, Fuel technology and the classification of coal. Proc. S. Wales Inst. Eng. 47 (1931), pp. 557-592.

Stopes, 1935. M.C. Stopes , On the petrology of banded bituminous. Fuel 14 (1935), pp. 413.

Taylor et al., 1998. G.H. Taylor, M. Teichmüller, A. Davis, C.F.K. Diessel, R. Littke and P. Robert , Organic Petrology. , Gebrüder Borntraeger, Stuttgart (1998) 703 pp. .

Walther, 1893-1894. J. Walther Einleitung in die Geologie als historische Wissenschaft vol. 3, Verlag von Gustav Fisher, Jena (1893-1894) 1055 pp. .

Weimer, 1960. R.J. Weimer , Upper Cretaceous stratigraphy, Rocky Mountain area. AAPG Bull. 44 (1960), pp. 1-20. 
Woodward and Callender, 1977. L.A. Woodward and J.F. Callendar, Tectonic framework of the San Juan Basin. In: New Mexico Geological Society Guidebook, 28th Field Conference (1977), pp. 209-212. 